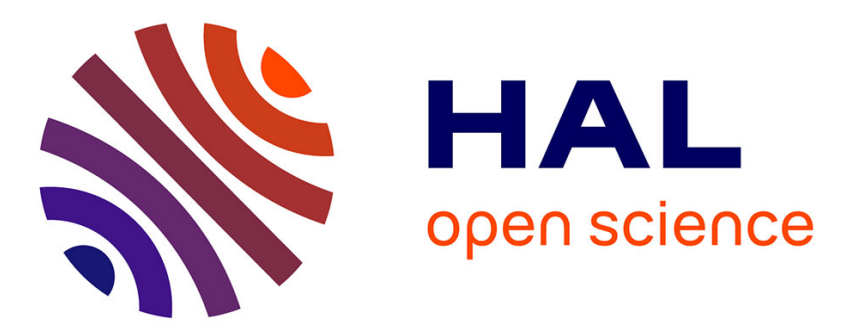

\title{
Sliding Mode Control with Adaptive Gain of Quadrotor with Rigid Manipulator
}

\author{
Hardy Azmir Anuar, Franck Plestan, Abdelhamid Chriette, Olivier \\ Kermorgant
}

\section{- To cite this version:}

Hardy Azmir Anuar, Franck Plestan, Abdelhamid Chriette, Olivier Kermorgant. Sliding Mode Control with Adaptive Gain of Quadrotor with Rigid Manipulator. International Conference on Advanced Robotics, Dec 2021, Ljubljana, Slovenia. hal-03445938

\section{HAL Id: hal-03445938 \\ https://hal.science/hal-03445938}

Submitted on 24 Nov 2021

HAL is a multi-disciplinary open access archive for the deposit and dissemination of scientific research documents, whether they are published or not. The documents may come from teaching and research institutions in France or abroad, or from public or private research centers.
L'archive ouverte pluridisciplinaire HAL, est destinée au dépôt et à la diffusion de documents scientifiques de niveau recherche, publiés ou non, émanant des établissements d'enseignement et de recherche français ou étrangers, des laboratoires publics ou privés. 


\title{
Sliding Mode Control with Adaptive Gain of Quadrotor with Rigid Manipulator
}

\author{
Hardy Azmir Anuar ${ }^{1}$, Franck Plestan ${ }^{2}$, Abdelhamid Chriette ${ }^{3}$ and Olivier Kermorgant ${ }^{4}$
}

\begin{abstract}
This paper addresses the problem of stabilizing the motion of an aerial manipulator composed of a Quadrotor and a manipulator with two degrees of freedom ( 2 dof $)$. The dynamic modeling of an aerial manipulator, like the one presented in this paper, shows that there is a strong physical coupling between the dynamics of the UAV and the manipulator arm. This coupling, plus external perturbations (e.g. wind gusts), considerably affect the stability of the drone's motion in flight which, consequently, affects the desired accuracy of the end effector with respect to its final task. To overcome this problem, we propose in this paper an approach based on an adaptive gain sliding mode control, a priory without the need to know its bounds, and to make the gain as small as possible but sufficient to overcome these disturbances due to both the arm motion and external uncertainties. The effectiveness of the controller and its robustness against noise are verified and analyzed using MATLAB/SIMULINK.
\end{abstract}

\section{INTRODUCTION}

Unmanned Aerial Vehicles (UAVs) have been used in a number of applications. In particular, UAVs are exploited in entertainment and surveillance applications, and research is in progress for their use in construction, inspection, and logistics industries. Recently, UAVs have been used in tasks such as grasping and manipulation [1], [2] as well as in cooperative transportation [3]. These are challenging conditions since UAVs is characterized by its unstable dynamics and in the presence of object creates significant coupling effects [4]. UAVs with rigid manipulator for aerial manipulations have been proposed in [5]

To accomplished manipulation task in the air, UAV needs to be equipped with a proper tools. Two most common solutions are either to mount a griper or multi fingered hand directly on the UAV, known as flying hand or to equip UAV with one or more robotic manipulator, hence the name unmanned aerial manipulator (UAM) [5]. With flying hand it is not possible to do more than pick and place operation which is not enough for manipulation task because the gripper cannot be moved independently from the UAV.

There are two approaches to address modeling and control problems [5] [6]. The first approach is an overall approach, in which the UAV and the robotic arm are considered a unified system as in [7]-[12]. This approach is quite difficult and complex. The second approach is to considers the UAV and the robotic arm as two separate independent systems as in [13]-[18], and then builds models and controllers separately.

\footnotetext{
The authors are with Ecole Centrale de Nantes, France.

${ }^{1}$ Hardy Azmir Anuar: Hardy. Anuarels2n.fr

${ }^{2}$ Franck Plestan: Franck.Plestan@ec-nantes.fr

${ }^{3}$ Abdelhamid Chriette: Abdelhamid. Chriette@ec-nantes. fr

${ }^{4}$ Olivier Kermorgant: Olivier.Kermorgant@ec-nantes.fr
}

The control of the motion of a drone equipped with a rigid arm is a difficult problem, since, on the one hand, the quadrotor is an under-actuated system, with two directions that are not directly actuated, and, on the other hand, the existence of an additional torque caused by the movement of the arm and which disturbs the stability and the accuracy of the positioning task of the full system \{drone+arm\}. Kim et al [8] developed an adaptive sliding mode controller with estimated uncertainty to cope with this disturbance. An experimental demonstrates satisfactory performance for picking up and delivering an object. As the movement of manipulator may disturb the attitude of UAV which affect flight stability and operation accuracy, Zhiyuan et al [19] proposed a fuzzy sliding mode controller with extended state observer for estimating disturbance. The fuzzy controller function is to eliminate the chattering effect of sliding mode control (SMC).

Wang et al [20] adopted to an improved active disturbance rejection control strategy to handled the disturbance from the manipulator and external environment on the robustness of the UAV controller. The strategy make use both an extended state observer and a dynamic centre of mass disturbance compensator for disturbance observation. Chen et al [21] discuss tracking control strategy due to internal and external disturbance. These disturbance are handled in two parts. In position controller using a robust sliding mode control and in attitude controller using an adaptive controller with disturbance observer.

Based on the compensation of the dynamic coupling between a UAV and a manipulator, Mimmo et al [22] proposed a control scheme that is able to let the endeffector to track a desired reference manoeuver while the UAV is maintained at a constant position. The manipulator is stabilised by compensating the UAV attitude whereas the joint torque is use in the computation of UAV control law. The interconnection of these two subsystems has been proven to be stable. Taking the equation of motion of a unified system in the form of a standard robot dynamics equation, Bulut et al [23] proposed a computed torque with PID controller to the unified system. In the present of interaction forces, the controller shows a robust behaviour and brings the UAV to its original position.

In this paper, in terms of dynamic modeling, we adopt the approach of considering the dynamic modeling of the UAV independently from that of the robotic arm. Model for a full system is builds based on the compensation of the coupling effect between UAV and robotic arm considered as external disturbances. 
Contribution of this paper is on the proposed control scheme which is based on adaptive gain sliding mode controller (SMCA). This controller is used in addition to approximate linearization for UAV and computed torque control of manipulator. With SMCA it is unnecessary to know the bound of uncertainties and the sliding mode gain will not be over estimate. It is feasible with the proposed scheme to achieve system stability, accuracy and robustness.

The rest of the paper is organized as follows. In Section II, the system modelling is introduced. The control law design is presented in Section III. Simulation results are presented in Section IV. Finally discussion of the results, conclusion and perspectives are presented in section $\mathrm{V}$.

\section{SYSTEM MODELLING}

This section introduce the modeling of UAM, starting from the classical modeling of multi-rotor. The addition of a manipulator arm, and its modeling, leads to a complete model for UAM.

Before deriving UAM dynamics, we define some reference frames. The inertial frame, also referred as world frame, is denoted $F_{W}$ and defined by its origin $O_{W}$ and three unit vectors along the main axes denoted $\left\{x_{W}, y_{W}, z_{W}\right\}$. The compact notation for this definition yields $F_{W}: O_{W}-$ $\left\{x_{W}, y_{W}, z_{W}\right\}$. The body frame of the multi-rotor is $F_{B}: O_{B}-\left\{x_{B}, y_{B}, z_{B}\right\}$, where $O_{B}$ is located at the center of mass $(\mathrm{CoM})$ of the UAV. $F_{0}$ is the base frame of manipulator attached to UAV body frame, while $F_{1}, F_{2}$ and $F_{e}$ are the frames of manipulator link-1, link-2 and end-effector, see Fig. 1 for illustration.

\section{A. Multi-rotor UAV Dynamics}

The position of $O_{B}$ expressed in $F_{W}$ is denoted by $\boldsymbol{\xi}=$ $\left[\begin{array}{lll}x & y & z\end{array}\right]^{T}$. The attitude of the UAV is defined by the three Euler angles of roll, pitch and yaw given by $\boldsymbol{\eta}=[\phi \theta \psi]^{T}$.

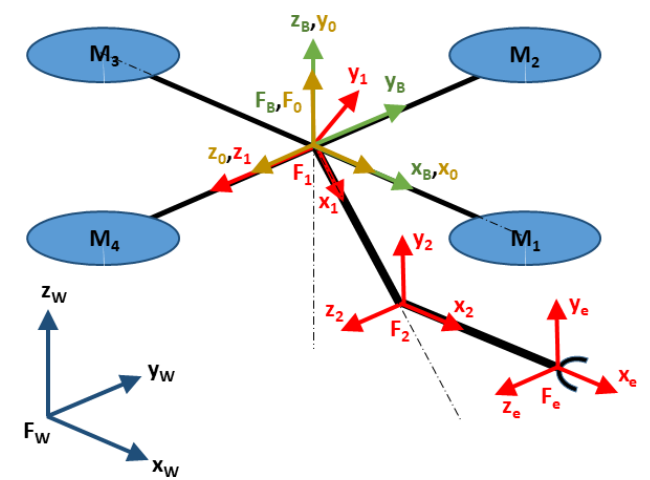

Fig. 1. UAV with a 2-dof manipulator arm.

The UAV dynamics can be derived from Newton Euler formulation with the following assumptions [24]:

- The structure is rigid and symmetrical such that the inertia matrix $I_{x x}=I_{y y}$.

- The centre of gravity coincide with body fixed frame.

- The propellers are rigid.

- Thrust and drag are proportional to the square of propellers speed.
By applying Newton-Euler equation [25], the dynamic model of UAV is given by:

$$
\begin{aligned}
\ddot{x} & =\frac{U_{1}}{m}(C \psi S \theta C \phi+S \psi S \phi) \\
\ddot{y} & =\frac{U_{1}}{m}(S \psi S \theta C \phi-C \psi S \phi) \\
\ddot{z} & =\frac{U_{1}}{m} C \theta C \phi-g \\
\ddot{\phi} & =\frac{U_{2}}{I_{x x}}+\frac{I_{y y}-I_{z z}}{I_{x x}} \dot{\theta} \dot{\psi} \\
\ddot{\theta} & =\frac{U_{3}}{I_{y y}}+\frac{I_{z z}-I x x}{I_{y y}} \dot{\phi} \dot{\psi} \\
\ddot{\psi} & =\frac{U_{4}}{I_{z z}}+\frac{I_{x x}-I_{y y}}{I_{z z}} \dot{\phi} \dot{\theta}
\end{aligned}
$$

in which $m$ is the mass of the drone, $S x=\sin (x)$ and $C x=\cos (x)$ while the control inputs $U_{1}, U_{2}, U_{3}$ and $U_{4}$ are the upward thrust, rolling torque, pitching torque and yawing torque respectively.

\section{B. Modelling of Manipulator}

The manipulator dynamics are established by the recursive Newton Euler (RNE) algorithm. The RNE algorithm as in [26] consists of two recursive computations: forward recursive and backward recursive. Detail dicussion of this algorithm can be found in [26] and [27].

1) Forward Recursive: Computes angular velocity $(\omega)$, angular acceleration $(\dot{\boldsymbol{\omega}})$ and linear acceleration $(\dot{\boldsymbol{v}})$ from base to the terminal link and consequently the joint force $\left(\boldsymbol{f}_{t}\right)$ and moment $\left(\boldsymbol{m}_{t}\right) . \boldsymbol{\omega}_{j}, \dot{\boldsymbol{\omega}}_{j}, \dot{\boldsymbol{v}}_{j}, \boldsymbol{f}_{t j}$ and $\boldsymbol{m}_{t j}$ on each link- $j$ are given as:

$$
\begin{aligned}
\boldsymbol{\omega}_{j} & =\boldsymbol{\omega}_{j-1}+\dot{\boldsymbol{q}}_{j} \boldsymbol{z}_{0} \\
\dot{\boldsymbol{\omega}}_{j} & =\dot{\boldsymbol{\omega}}_{j-1}+\ddot{\boldsymbol{q}}_{j} \boldsymbol{z}_{0}+\boldsymbol{\omega}_{j-1} \times \dot{\boldsymbol{q}}_{j} \boldsymbol{z}_{0} \\
\dot{\boldsymbol{\nu}}_{j} & =\dot{\boldsymbol{\nu}}_{j-1}+\dot{\boldsymbol{\omega}}_{j-1} \times \boldsymbol{L}_{j-1}+\boldsymbol{\omega}_{j-1} \times\left(\boldsymbol{\omega}_{j-1} \times \boldsymbol{L}_{j}\right) \\
\boldsymbol{f}_{t j} & =M_{j} \dot{\boldsymbol{v}}_{j}+\dot{\boldsymbol{\omega}}_{j} \times \boldsymbol{M} \boldsymbol{S}_{j}+\boldsymbol{\omega}_{j} \times\left(\boldsymbol{\omega}_{j} \times \boldsymbol{M} \boldsymbol{S}_{j}\right) \\
\boldsymbol{m}_{t j} & =\boldsymbol{I}_{o j} \dot{\boldsymbol{\omega}}_{j}+\boldsymbol{M} \boldsymbol{S}_{j} \times \dot{\boldsymbol{v}}_{j}+\boldsymbol{\omega}_{j} \times\left(\boldsymbol{I}_{o j} \boldsymbol{\omega}_{j}\right)
\end{aligned}
$$

where $\boldsymbol{q}_{j}$ and $\boldsymbol{z}_{0}$ are the joint position vector and a unit vector along z-axis respectively. $M_{j}, \boldsymbol{M} \boldsymbol{S}_{j}$ and $\boldsymbol{L}_{j}$ are the mass, first moment and position vector of link- $j$.

2) Backward Recursive: Computes the resultant force $(\boldsymbol{f})$ and moment $(\boldsymbol{m})$ from the terminal link to the base and consequently the joint torque $(\boldsymbol{\Gamma}) .\left(\boldsymbol{f}_{j}\right)$ and $\left(\boldsymbol{m}_{j}\right)$ for each link- $j$ are given as:

$$
\begin{aligned}
\boldsymbol{f}_{j} & =\boldsymbol{f}_{t j}+\boldsymbol{f}_{j+1}+\boldsymbol{f}_{e j} \\
\boldsymbol{m}_{j} & =\boldsymbol{m}_{t j}+\boldsymbol{m}_{j+1}+\boldsymbol{L}_{j+1} \times \boldsymbol{f}_{j+1}+\boldsymbol{m}_{e j}
\end{aligned}
$$

$\boldsymbol{f}_{e j}$ and $\boldsymbol{m}_{e j}$ represent the force and moment exerted by joint $-j$ on the environment. Finally the joint torque can be obtained as:

$$
\Gamma_{j}=\boldsymbol{m}_{j}^{T} \boldsymbol{z}_{0}+\Gamma_{f j}
$$

$\Gamma_{f j}$ denotes the friction torque of joint $-j$. If RNE are executed symbolically it will eventually form an expression of manipulator dynamic as:

$$
\Gamma=M(q) \ddot{q}+C(q, \dot{q}) \dot{q}+Q(q)
$$


where $\Gamma$ is the joint torque, $\boldsymbol{M}(\boldsymbol{q})$ is the symmetric and positive definite inertia matrix, $\boldsymbol{C}(\boldsymbol{q}, \dot{\boldsymbol{q}})$ is the vector of Coriolis and centrifugal torques and finally, $Q(q)$ is the vector of gravity torques.

\section{Modelling of UAM}

Modelling of UAM is based on the coupling effect of the manipulator arm on UAV as external disturbances and vice versa as in [17] and [18]. The configuration of the manipulator arm in relation to the inertial fixed frame, $F_{W}$ is given as rotation matrix:

$$
{ }^{0} \boldsymbol{R}_{W}={ }^{0} \boldsymbol{R}_{B}{ }^{B} \boldsymbol{R}_{W}
$$

where

$$
{ }^{0} \boldsymbol{R}_{B}=\left[\begin{array}{ccc}
1 & 0 & 0 \\
0 & 0 & 1 \\
0 & -1 & 0
\end{array}\right]
$$

while ${ }^{B} \boldsymbol{R}_{W}$ a rotation matrix used to describe the position in the inertial frame with respect to UAV body frame.

For a floating base the initial velocity and acceleration for the RNE algorithm is given as follows.

$$
\begin{aligned}
\dot{\boldsymbol{\nu}}_{0} & ={ }^{0} \boldsymbol{R}_{W}\left(\ddot{\boldsymbol{\xi}}_{W}+\left[\begin{array}{lll}
0 & 0 & -g
\end{array}\right]^{T}\right) \\
\boldsymbol{\omega}_{0} & ={ }^{0} \boldsymbol{R}_{B} \boldsymbol{\omega}_{B} \\
\dot{\boldsymbol{\omega}}_{0} & ={ }^{0} \boldsymbol{R}_{B} \dot{\boldsymbol{\omega}}_{B}
\end{aligned}
$$

This velocity and acceleration are transmitted from one link to another and result in an additional resultant torque on joint- $j$.

The force, $\boldsymbol{f}_{W}$, and torque, $\boldsymbol{\tau}_{B}$, applied to UAV as external disturbance is then obtained from force, $\boldsymbol{f}_{1}$, and moment, $\boldsymbol{m}_{1}$, at joint- 1 as:

$$
\begin{aligned}
\boldsymbol{f}_{W} & ={ }^{W} \boldsymbol{R}_{0}{ }^{0} \boldsymbol{R}_{1} \boldsymbol{f}_{1} \\
\boldsymbol{\tau}_{B} & ={ }^{B} \boldsymbol{R}_{0}{ }^{0} \boldsymbol{R}_{1} \boldsymbol{m}_{1}
\end{aligned}
$$

This coupling effect are the model coupling algorithm of coupled system as shown in figure 2. Consequently from (1) to (6) the dynamic equation of UAV due to coupling effect is then given as:

$$
\begin{aligned}
\ddot{x}_{u a m} & =\ddot{x}+\left(f_{W . x} / m\right) \\
\ddot{y}_{u a m} & =\ddot{y}+\left(f_{W . y} / m\right) \\
\ddot{z}_{u a m} & =\ddot{z}+\left(f_{W . z} / m\right) \\
\ddot{\phi}_{u a m} & =\ddot{\phi}+\left(\tau_{B . \phi} / I_{x x}\right) \\
\ddot{\theta}_{u a m} & =\ddot{\theta}+\left(\tau_{B . \theta} / I_{y y}\right) \\
\ddot{\psi}_{u a m} & =\ddot{\psi}+\left(\tau_{B . \psi} / I_{z z}\right)
\end{aligned}
$$

\section{Inverse Kinematic}

An inverse kinematic block shown in figure 2 produces reference trajectory for $\operatorname{UAV}\left(\boldsymbol{\xi}_{r}, \boldsymbol{\psi}_{r}\right)$ and manipulator $\left(\ddot{\boldsymbol{q}}_{r}, \dot{\boldsymbol{q}}_{r}, \boldsymbol{q}_{r}\right)$ from the desired end effector trajectory $\left(\dot{\boldsymbol{\xi}}_{T . d}, \boldsymbol{\xi}_{T . d}, \dot{\boldsymbol{\eta}}_{T . d}, \boldsymbol{\eta}_{T . d}\right)$. The generation of reference trajectory from inverse kinematic algorithm is discussed in details in [9], [28] and [29].
The linear velocity, $\dot{\boldsymbol{\xi}}_{T}$, and angular velocity, $\boldsymbol{\omega}_{T}$, of the end effector can be computed as follows.

$$
\begin{aligned}
\dot{\boldsymbol{\xi}}_{T} & =\dot{\boldsymbol{\xi}}_{B}-\boldsymbol{S}\left({ }^{W} \boldsymbol{R}_{B} \boldsymbol{\xi}_{e}\right) \boldsymbol{\omega}_{B}+{ }^{W} \boldsymbol{R}_{B} \dot{\boldsymbol{\xi}}_{e} \\
\boldsymbol{\omega}_{T} & =\boldsymbol{\omega}_{B}+{ }^{W} \boldsymbol{R}_{B} \boldsymbol{\omega}_{e}
\end{aligned}
$$

where $\boldsymbol{S}($.$) is a (3 \times 3)$ skew symmetric operator, $\boldsymbol{\xi}_{e}$ is the position vector of $F_{e}$ expressed in $F_{B}, \boldsymbol{\omega}_{B}$ is the angular velocity of $F_{B}$ and $\boldsymbol{\omega}_{e}$ is the angular velocity of $F_{e}$. If the attitude is express in the form of yaw-pitch-roll angle, $\boldsymbol{\eta}=$ $\left[\begin{array}{lll}\psi & \theta\end{array}\right]$, then equation (29) and (30) can be arranged as:

$$
\boldsymbol{T}_{T}\left(\boldsymbol{\eta}_{T}\right) \dot{\boldsymbol{x}}_{T}=\boldsymbol{J}_{B}\left(\boldsymbol{\eta}_{B}, \boldsymbol{q}\right) \dot{\boldsymbol{x}}_{B}+\boldsymbol{J}_{e}\left(\boldsymbol{\eta}_{B}, \boldsymbol{q}\right) \dot{\boldsymbol{q}}
$$

where

$$
\begin{aligned}
& \dot{\boldsymbol{x}}_{T}=\left[\begin{array}{c}
\dot{\boldsymbol{\xi}}_{T} \\
\dot{\boldsymbol{\eta}}_{T}
\end{array}\right] \\
& \dot{\boldsymbol{x}}_{B}=\left[\begin{array}{c}
\dot{\boldsymbol{\xi}}_{B} \\
\dot{\boldsymbol{\eta}}_{B}
\end{array}\right] \\
& \boldsymbol{T}_{T}\left(\boldsymbol{\eta}_{T}\right)=\left[\begin{array}{cc}
\boldsymbol{I}_{3} & \boldsymbol{O}_{3} \\
\boldsymbol{O}_{3} & \boldsymbol{T}\left(\boldsymbol{\eta}_{T}\right)
\end{array}\right] \\
& \boldsymbol{J}_{B}\left(\boldsymbol{\eta}_{B}, \boldsymbol{q}\right)=\left[\begin{array}{cc}
\boldsymbol{I}_{3} & -\boldsymbol{S}\left({ }^{W} \boldsymbol{R}_{B} \boldsymbol{\xi}_{e}\right) \boldsymbol{T}\left(\boldsymbol{\eta}_{B}\right) \\
\boldsymbol{O}_{3} & \boldsymbol{T}\left(\boldsymbol{\eta}_{B}\right)
\end{array}\right] \\
& \boldsymbol{J}_{e}\left(\boldsymbol{\eta}_{B}, \boldsymbol{q}\right)=\left[\begin{array}{cc}
W \boldsymbol{R}_{B} & \boldsymbol{O}_{3} \\
\boldsymbol{O}_{3} & { }^{W} \boldsymbol{R}_{B}
\end{array}\right] \boldsymbol{J}(\boldsymbol{q})
\end{aligned}
$$

and $\boldsymbol{T}($.$) is the transformation matrix that relates the angular$ velocity to the Euler rate, $\boldsymbol{J}(\boldsymbol{q})$ is the Jacobian matrix of joint velocity, $\dot{\boldsymbol{q}}$ and, $\boldsymbol{I}_{3}$ and $\boldsymbol{O}_{3}$ denote $(3 \times 3)$ identity and null matrices respectively.

Since the position and yaw angle are usually the control variable of UAV while roll and pitch are used for intermediate control, $\boldsymbol{x}_{B}$ can be rewrite as.

$$
\boldsymbol{x}_{B}=\left[\begin{array}{l}
\boldsymbol{\alpha}_{B} \\
\boldsymbol{\sigma}_{B}
\end{array}\right], \boldsymbol{\alpha}_{B}=\left[\begin{array}{l}
\boldsymbol{\xi}_{B} \\
\psi_{B}
\end{array}\right], \boldsymbol{\sigma}_{B}=\left[\begin{array}{l}
\theta_{B} \\
\phi_{B}
\end{array}\right]
$$

Thus from (31), the differential kinematics becomes:

$$
\begin{aligned}
\dot{\boldsymbol{x}}_{T}= & \boldsymbol{T}_{T}\left(\boldsymbol{\eta}_{T}\right)^{-1}\left[\boldsymbol{J}_{\alpha}\left(\boldsymbol{\zeta}, \boldsymbol{\sigma}_{B}\right) \dot{\boldsymbol{\alpha}}_{B}+\boldsymbol{J}_{\sigma}\left(\boldsymbol{\zeta}, \boldsymbol{\sigma}_{B}\right) \dot{\boldsymbol{\sigma}}_{B}\right. \\
& \left.+\boldsymbol{J}_{e}\left(\boldsymbol{\zeta}, \boldsymbol{\sigma}_{B}\right) \dot{\boldsymbol{q}}\right] \\
= & \boldsymbol{T}_{T}\left(\boldsymbol{\eta}_{T}\right)^{-1}\left[\boldsymbol{J}_{\zeta}\left(\boldsymbol{\zeta}, \boldsymbol{\sigma}_{B}\right) \dot{\boldsymbol{\zeta}}_{B}+\boldsymbol{J}_{\sigma}\left(\boldsymbol{\zeta}, \boldsymbol{\sigma}_{B}\right) \dot{\boldsymbol{\sigma}}_{B}\right]
\end{aligned}
$$

where $\boldsymbol{J}_{\alpha}$ is the first 4 columns of $\boldsymbol{J}_{B}, \boldsymbol{J}_{\sigma}$ is the last 2 columns of $\boldsymbol{J}_{B}, \boldsymbol{J}_{\zeta}=\left[\boldsymbol{J}_{\alpha} \boldsymbol{J}_{e}\right]$ and $\boldsymbol{\zeta}=\left[\begin{array}{l}\boldsymbol{\alpha}_{B} \\ \boldsymbol{q}\end{array}\right]^{T}$.

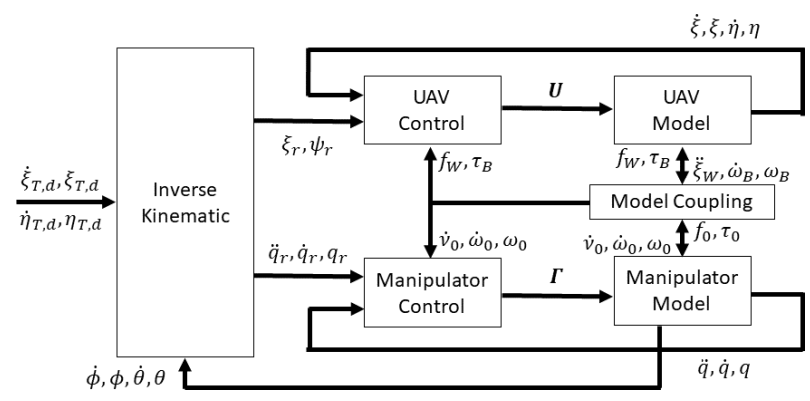

Fig. 2. Full block diagram of a coupled system. 


\section{CONTROL DESIGN}

\section{A. Closed Loop Inverse Kinematic}

Recalling differential kinematic (37) and considering the closed-loop inverse kinematics algorithm [30], the computation of the reference trajectories for UAV and manipulator arm as follows:

$$
\begin{aligned}
\dot{\boldsymbol{\zeta}}_{r}= & \boldsymbol{J}_{\zeta}\left(\boldsymbol{\zeta}_{r}, \boldsymbol{\sigma}_{B}\right)^{-1} \boldsymbol{T}_{T}\left(\boldsymbol{\eta}_{T . r}\right)\left(\dot{\boldsymbol{x}}_{T . d}+\boldsymbol{K} \boldsymbol{e}\right) \\
& -\boldsymbol{J}_{\zeta}\left(\boldsymbol{\zeta}_{r}, \boldsymbol{\sigma}_{B}\right)^{-1} \boldsymbol{J}_{\sigma}\left(\boldsymbol{\zeta}_{r}, \boldsymbol{\sigma}_{B}\right) \dot{\boldsymbol{\sigma}}_{B}
\end{aligned}
$$

where $\boldsymbol{K}$ is a symmetric positive definite gain matrix, $\boldsymbol{e}$ is the kinematic error defined by $\boldsymbol{e}=\boldsymbol{x}_{T . d}-\boldsymbol{x}_{T . r}$ and $\boldsymbol{x}_{T . r}$ is the end effector pose using direct kinematics from computed value $\zeta_{r}$ and measured value $\boldsymbol{\sigma}_{B}$.

\section{B. UAV Control}

UAV control system is designed by a hierarchical manner with inner and outer control loop as shown in figure 3 .

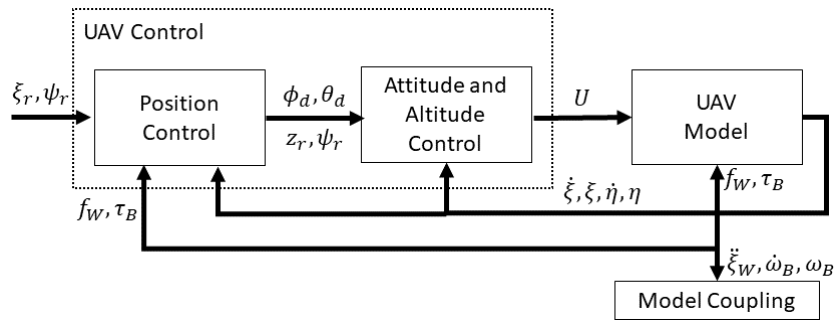

Fig. 3. UAV hierarchical control.

By applying small angle approximation with near hover state, the control synthesis for inner loop attitude and altitude control are derived from (25) to (28) as follows:

$$
\begin{aligned}
& U_{1}=m(\ddot{z}+g)-f_{W . z} \\
& U_{2}=I_{x x} \ddot{\phi}-\tau_{B . \phi} \\
& U_{3}=I_{y y} \ddot{\theta}-\tau_{B . \theta} \\
& U_{4}=I_{z z} \ddot{\psi}-\tau_{B . \psi}
\end{aligned}
$$

Outer control loop gives desired roll and pitch angles derived from (23) and (24) as:

$$
\left[\begin{array}{l}
\phi_{d} \\
\theta_{d}
\end{array}\right]=\frac{1}{g}\left[\begin{array}{cc}
S \psi & -C \psi \\
C \psi & S \psi
\end{array}\right]\left[\begin{array}{l}
\ddot{x}-\frac{f_{W \cdot x}}{m} \\
\ddot{y}-\frac{f_{W \cdot y}}{m}
\end{array}\right]
$$

where $\ddot{x}=\nu_{x}, \ddot{y}=\nu_{y}, \ddot{z}=\nu_{z}, \ddot{\phi}=\nu_{\phi}, \ddot{\theta}=\nu_{\theta}$ and $\ddot{\psi}=$ $\nu_{\psi}$ are the control variables. In the sequel, the approaches used for the control input $\nu($.$) are PID feedback control and$ sliding mode control (SMC).

\section{Manipulator Control}

When the task requires high dynamic accuracy, it is necessary to take into account manipulator dynamic model via a computed torque control technique [27] [31] given by control input:

$$
\Gamma=M(q) \nu_{q}+C(q, \dot{q}) \dot{q}+Q(q)
$$

Computed torque control as shown in Figure 4 consists of inner nonlinear compensation loop of $\boldsymbol{M}(\boldsymbol{q}), \boldsymbol{C}(\boldsymbol{q}, \dot{\boldsymbol{q}})$ and $Q(q)$ and an outer loop for the control input, $\nu_{q}$.

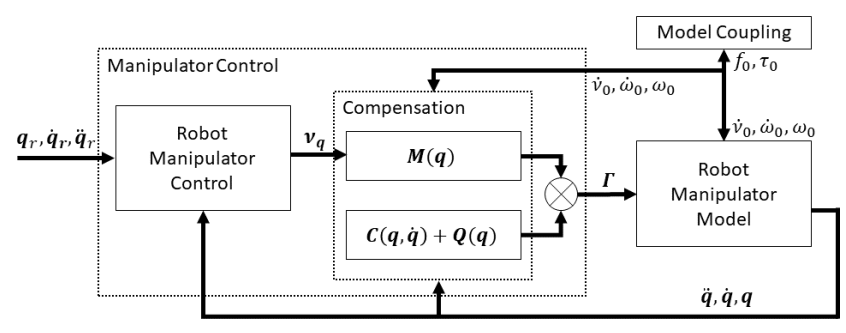

Fig. 4. Manipulator computed torque control.

Including this control law into the dynamical model of the manipulator (15) gives:

$$
\nu_{q}=\ddot{q}
$$

As previously, the approach for the outer loop control is either PID feedback control or SMC.

\section{Sliding Mode Control}

In the presence of inconsistency that occurs from external disturbances and uncertain in model dynamics, robust control methods are required. Robust control such as sliding mode controller (SMC) has been designed to control such systems [32]-[34]. SMC design consists of two phase. First is the design of sliding surface and secondly is the design of control law to force system trajectories onto sliding surface and holds onto it in spite of the presence of inconsistency.

1) Sliding Surface Design: Consider the following state variables for position $\left(x_{1}=x\right)$ and velocity $\left(x_{2}=\dot{x}_{1}\right)$

$$
\begin{aligned}
& \dot{x}_{1}=x_{2} \\
& \dot{x}_{2}=f(x)+g(x) \nu
\end{aligned}
$$

where $\nu$ is the control input, $f(x)$ is the external disturbance and $g(x) \neq 0 . f(x)$ and $g(x)$ are smooth uncertain functions and are bounded. The control objective is to drive the states variables to zero.

Now define a sliding surface function, $S$, such that when $S=0$ control objective is achieved. The most typical function is linear time-invariant differential equation

$$
S=x_{2}+\lambda x_{1}, \quad \lambda>0
$$

When $S=0$

$$
x_{2}=-\lambda x_{1}
$$

then both $x_{1}$ and $x_{2}$ will converge to zero asymptotically [32]. In the presence of bounded disturbance, $S$ must be forced to zero in finite time by means of control law. 
2) Control Law Design: To design the control law, a candidate Lyapunov function is introduced:

$$
V=\frac{1}{2} S^{2}
$$

To ensure the asymptotic convergence of $S$, time derivative of (50) must be negative definite.

$$
\dot{V}=S \dot{S}<0
$$

This inequality is the sliding condition. Then:

$$
\begin{aligned}
\dot{S} & =\dot{x}_{2}+\lambda \dot{x}_{1} \\
& =f(x)+g(x) \nu+\lambda x_{2}
\end{aligned}
$$

and

$$
\dot{V}=S \dot{S}=S\left[\frac{f(x)+\lambda x_{2}}{g(x)}+\nu\right]
$$

Defining an upper bound as:

$$
\rho \geqslant\left|\frac{f(x)+\lambda x_{2}}{g(x)}\right|,
$$

and the control law as:

$$
\nu=-K \operatorname{sign}(S)
$$

then, one gets

$$
\begin{aligned}
\dot{V} & \leqslant|S| \rho-S K \operatorname{sign}(S) \\
& \leqslant|S| \rho-|S| K \\
& \leqslant-|S|(K-\rho)
\end{aligned}
$$

If $K>\rho$, then condition (51) is satisfied.

The main drawback of SMC is a phenomenon called chattering [35] [34]. Chattering causes high frequency oscillations on the control input that leads to reduced accuracy and increases actuator mechanical wear and tear [34]. Chattering effect can be reduced by replacing the sign function in (55) by an approximate continuous function in a vicinity of the origin of sliding surface [36], such as a saturation function given by:

$$
\operatorname{sat}(S, \epsilon)= \begin{cases}\operatorname{sign}(S) & \text { if }|S|>\epsilon \\ \frac{S}{\epsilon} & \text { if }|S| \leqslant \epsilon\end{cases}
$$

where $\epsilon>0$.

Another drawback is that SMC requires the knowledge of uncertainties bound which often leads to over-estimated bounds. A way to counteract this drawback is to ensure a dynamical adaptation of the control gain to achieve sliding mode condition and sufficient to overcome the unknown uncertainties. Therefore, a value of $K$ for a dynamically tuned adaptive gain sliding mode control (SMCA) is given as [35]:

$$
\dot{K}= \begin{cases}\bar{K} \cdot \operatorname{sign}(|S|-\mu) & \text { if } K>\alpha \\ \alpha & \text { if } K \leqslant \alpha\end{cases}
$$

with $\bar{K}>0, K_{0}>0, \mu>0$ and $\alpha>0$ very small. The parameter $\alpha$ ensures that $K$ is always positive. $\bar{K}$ determine the dynamics of the gain while $\mu$ acts on the accuracy. For adaptive gain law (58), the gain $K$ will increase at constant rate until $|S|<\mu$; then, the gain decreases and is kept at the smallest level as long as $S$ remain within $\mu$. With this adaptive control gain, the knowledge of uncertainties bound is not required. Furthermore, the adaptation gain allows to have the "just sufficient" gain that reduces the chattering effect.

\section{SIMULATION}

To validate the proposed modelling and control, simulations have been run considering the following parameters for the UAV. Mass of UAV, rotor distance from CoM, thrust coefficient and torque coefficient are $2 \mathrm{~kg}, 0.25 \mathrm{~m}$, $3 \times 10^{-5} \mathrm{Ns}^{2}$ and $7.5 \times 10^{-7} \mathrm{Nms}^{2}$ respectively. Its inertia matrix $=\operatorname{diag}([1.24,1.24,2.48])$. The UAV is equipped with 2-dof revolute manipulator arm with both mass, link length and moments of inertia about their joints are $0.1 \mathrm{~kg}, 0.25 \mathrm{~m}$ and $0.0021 \mathrm{kgm}^{2}$ respectively. The simulation model is built using Matlab/Simulink software. In this paper, comparison is made between PID, SMC and SMCA controllers. The controllers parameters for PID, SMC and SMCA are shown in table (I), (II) and (III) below.

TABLE I

PID PARAMETERS

\begin{tabular}{cccc} 
Parameter & $K_{p}$ & $K_{i}$ & $K_{d}$ \\
\hline Quad Pos X & 8.5 & 1.2 & 2.9 \\
Quad Pos Y & 8.5 & 1.2 & 2.9 \\
Quad Pos Z & 27 & 17.5 & 10.4 \\
Quad Roll & 150 & 230 & 24.5 \\
Quad Pitch & 150 & 230 & 24.5 \\
Quad Yaw & 27 & 17.5 & 10.4 \\
Joint Q1 & 40 & 31.62 & 12.65 \\
Joint Q2 & 40 & 31.62 & 12.65 \\
\hline
\end{tabular}

TABLE II SMC PARAMETERS

\begin{tabular}{cccc} 
Parameter & $\lambda$ & $K$ & $\epsilon$ \\
\hline Quad Pos X & 9 & 1.5 & 1 \\
Quad Pos Y & 9 & 1.5 & 1 \\
Quad Pos Z & 30 & 7 & 0.1 \\
Quad Roll & 40 & 5 & 0.5 \\
Quad Pitch & 40 & 5 & 0.5 \\
Quad Yaw & 70 & 7 & 0.1 \\
Joint Q1 & 2 & 50 & 0.1 \\
Joint Q2 & 12 & 300 & 1 \\
\hline
\end{tabular}


TABLE III

ADAPTIVE GAIN SMC PARAMETERS

\begin{tabular}{ccccccc} 
Parameter & $\lambda$ & $K_{0}$ & $\bar{K}$ & $\mu$ & $\epsilon$ & $\alpha$ \\
\hline Quad Pos X & 9 & 0.5 & 0.2 & 0.2 & 1 & 0.01 \\
Quad Pos Y & 9 & 0.5 & 0.2 & 0.2 & 1 & 0.01 \\
Quad Pos Z & 30 & 0.5 & 1 & 0.001 & 0.01 & 0.01 \\
Quad Roll & 40 & 0.5 & 0.5 & 0.01 & 0.5 & 0.01 \\
Quad Pitch & 40 & 0.5 & 2 & 0.01 & 0.8 & 0.01 \\
Quad Yaw & 70 & 0.5 & 1 & 0.0005 & 0.01 & 0.01 \\
Joint Q1 & 2 & 0.5 & 5 & 0.001 & 0.1 & 0.01 \\
Joint Q2 & 12 & 0.5 & 20 & 0.001 & 0.6 & 0.01 \\
\hline
\end{tabular}

A helical motion trajectory is used to evaluate the end effector tracking capability. This test is conducted either with or without introduction of external force on the system. This trajectory simulates all the translational and rotational motion simultaneously.

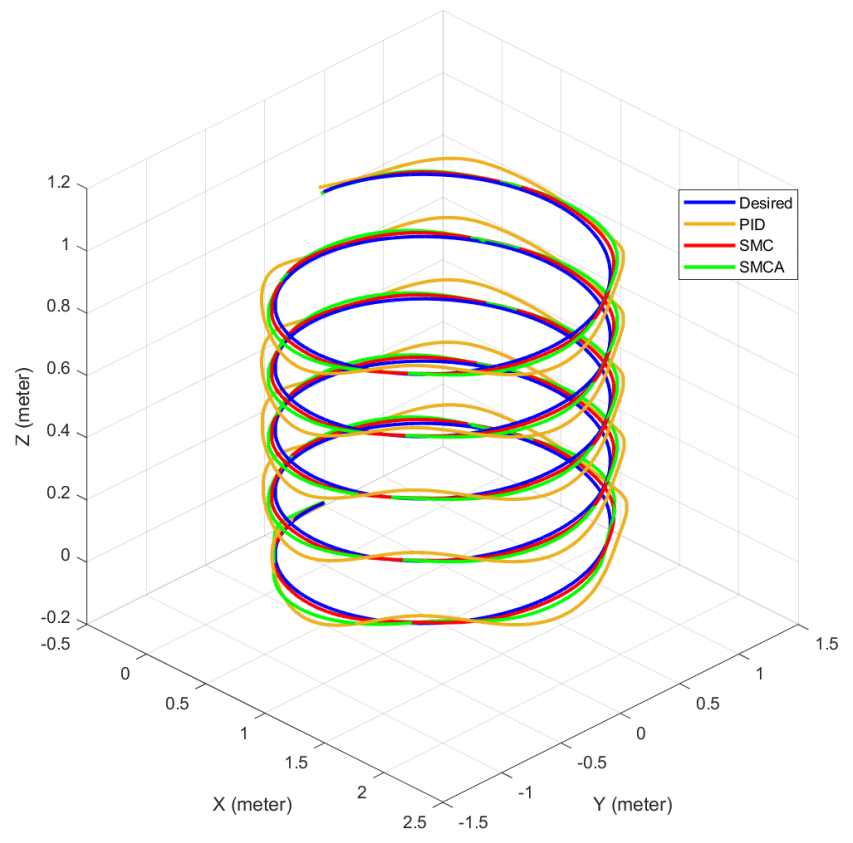

Fig. 5. 3D Trajectory of end effector.
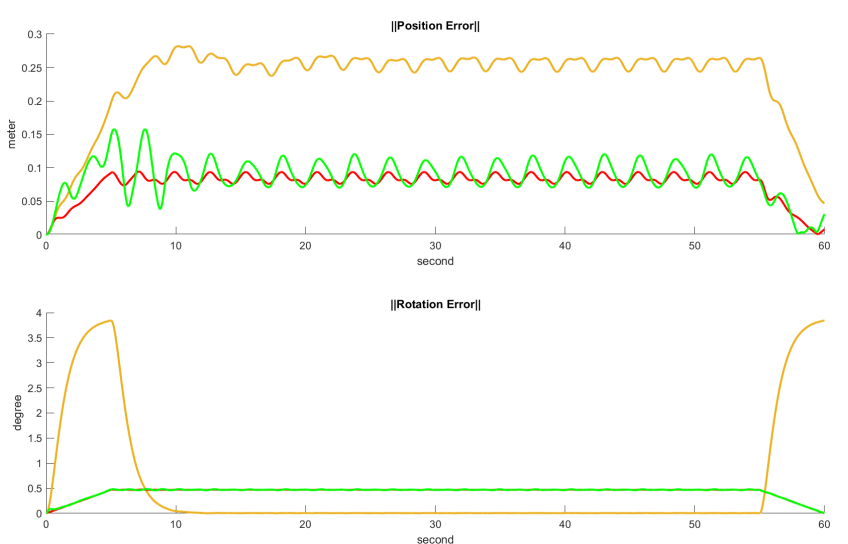

Fig. 6. End effector trajectory error.

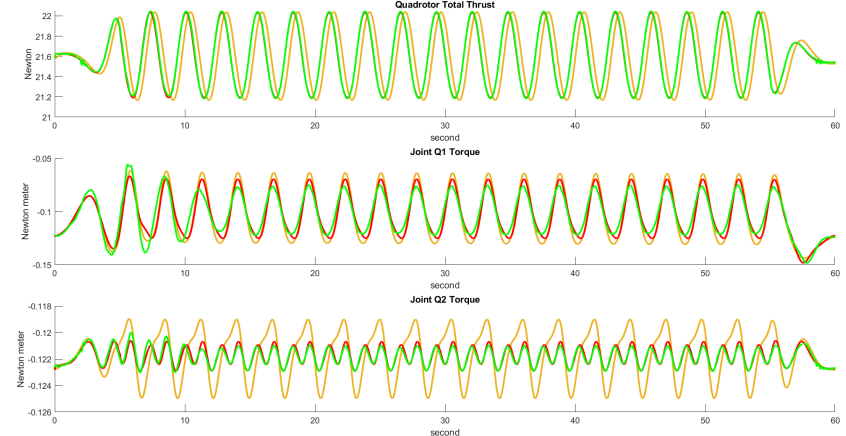

Fig. 7. End effector force and torque.

Two values are extracted from the simulations:

- the end effector position error, as the Euclidean distance between the desired and the estimated position error; and

- the end effector rotation error, as the absolute value of the error angle as:

$$
\theta_{e}=\left|\arccos \left(\left(\operatorname{tr}\left(\widehat{W}^{T}{ }^{T}{ }^{W} \boldsymbol{R}_{T}\right)-1\right) / 2\right)\right|
$$

where ${ }^{W} \boldsymbol{R}_{T}$ and $\widehat{W}^{W} \boldsymbol{R}_{T}^{T}$ are respectively the true and estimated end effector rotation matrices.

Figure 5 shows the end effector motion tracking comparison between PID (yellow), SMC (red), SMCA (green) with respect to the desired trajectory (blue) in the nominal case (no perturbation, no uncertainty). (A same colour scheme is used for all graph.) It shows the ability of sliding mode control to force the closed-loop system trajectory close to the desired trajectory. A nominal case tracking error is shown in figure 6 for the three controllers (the lower the value, the better the performance). It is clear that SMC and SMCA allow a smaller steady state error in position control. The rotation RMSE value of both SMC and SMCA is 0.47 as compared to PID of 0.51 . The error variation is also less so it is more stable. This error shows that sliding mode either with or without adaptive gain has more accurate and stable tracking performance. Furthermore, a small variation of torque for sliding mode (figure 7 - especially for adaptive one) will have less actuator mechanical wear and tear.

\section{A. With Disturbance}

1) Disturbance on UAV: The proposed controller schemes are now tested with external disturbance of $1 \mathrm{~N}$ introduced along $x$-axis of inertial frame while UAV tracks the trajectory. Figure 8 shows the end effector tracking error in the present of this disturbance. It shows that SMC and SMCA controller has become a little unstable but still keep their performances.

2) Disturbance on end effector: The proposed controller is also tested for mass loading by introducing a weight of $1 \mathrm{~N}$ at the tip of end effector. Figure 9 shows the tracking error of end effector with this disturbance. It shows that both sliding mode controllers are able to follow the trajectory in spite of this disturbance. As the weight cause a pitching torque it leads to rotation error. A close up view of the rotation 

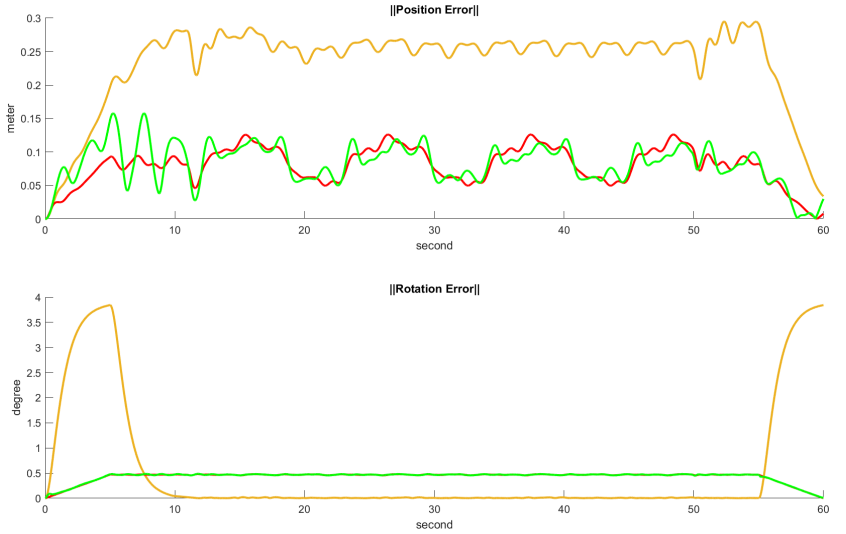

Fig. 8. End effector trajectory error with disturbance on UAV.

error (figure 10) shows that SMCA error (green) gradually decreased from around $2^{\circ}$ to about $0.5^{\circ}$ while SMC error (red) remains at around $2^{\circ}$. This demonstrate that adaptive gain of SMCA is working and it has a better error adaptation than SMC.
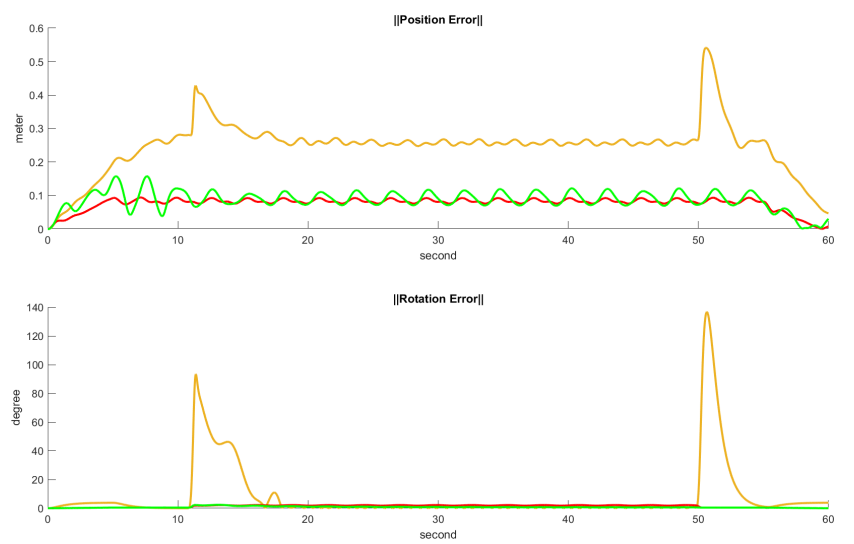

Fig. 9. End effector trajectory error with disturbance on end effector.

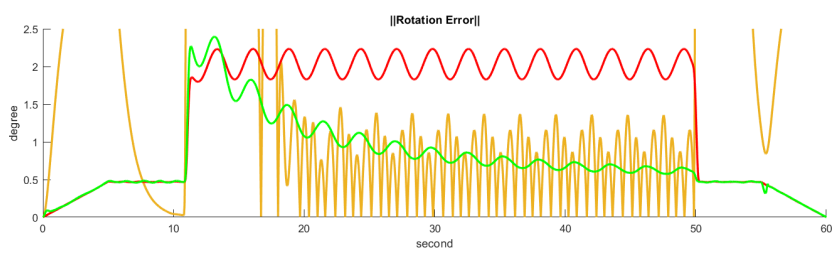

Fig. 10. End effector trajectory rotation error close up.

\section{CONCLUSion}

The normalise root mean square of tracking error (figure 11) shows SMCA though with a small gain $K$, has a comparable position control as SMC. Introduction of disturbance on UAV results in additional position error while a mass loading on end effector contribute to additional rotation error. While the variation of UAV force and joint torque (figure 12) for both sliding mode control is much less than PID, which reflect a stable actuator control. This simulation shows that the proposed adaptive gain sliding mode control even without the knowledge of uncertainty bound and with gain as small as possible able to overcome the disturbance from the arm motion and external force. It is shown the proposed scheme able to achieve system stability, accuracy and robustness.
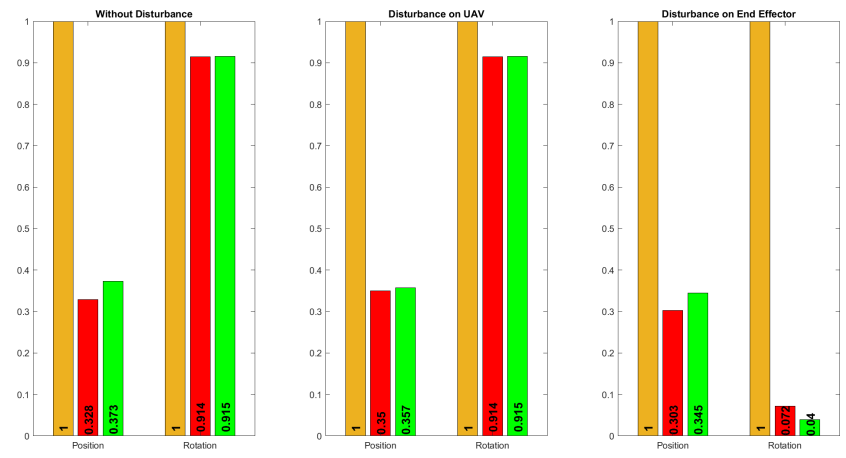

Fig. 11. End Effector normalize root mean square error.
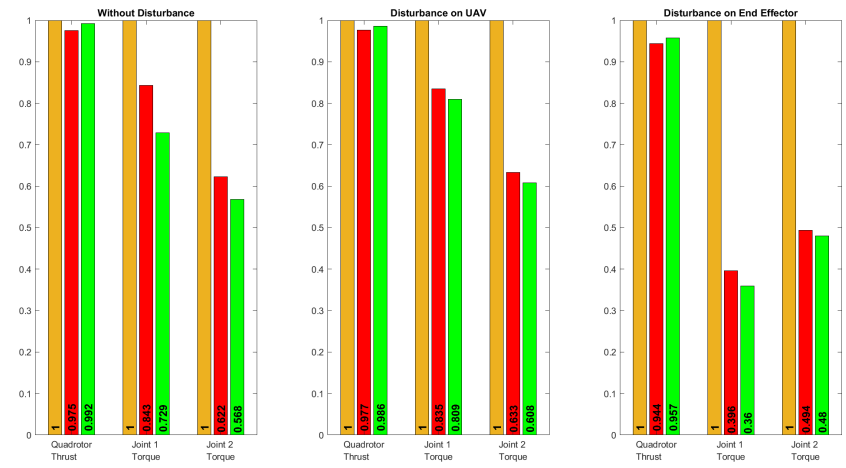

Fig. 12. End Effector force and torque variation. 


\section{REFERENCES}

[1] R. Spica, A. Franchi, G. Oriolo, H. H. Bülthoff, and P. R. Giordano, "Aerial grasping of a moving target with a quadrotor uav," in 2012 IEEE/RSJ International Conference on Intelligent Robots and Systems, 2012, pp. 4985-4992.

[2] H. B. Khamseh, F. Janabi-Sharifi, and A. Abdessameud, "Aerial manipulation-a literature survey," Robotics and Autonomous Systems, vol. 107, pp. 221-235, 2018.

[3] I. Maza, K. Kondak, M. Bernard, and A. Ollero, "Multi-uav cooperation and control for load transportation and deployment," Journal of Intelligent and Robotic Systems, vol. 57, pp. 417-449, 2010.

[4] P. E. I. Pounds, D. R. Bersak, and A. M. Dollar, "Grasping from the air: Hovering capture and load stability," in 2011 IEEE International Conference on Robotics and Automation, 2011, pp. 2491-2498.

[5] F. Ruggiero, V. Lippiello, and A. Ollero, "Aerial manipulation: A literature review," IEEE Robotics and Automation Letters, vol. 3, no. 3, pp. 1957-1964, 2018.

[6] X. Ding, P. Guo, K. Xu, and Y. Yu, "A review of aerial manipulation of small-scale rotorcraft unmanned robotic systems," Chinese Journal of Aeronautics, vol. 32, no. 1, pp. 200 - 214, 2019.

[7] V. Lippiello and F. Ruggiero, "Cartesian impedance control of a uav with a robotic arm," IFAC Proceedings Volumes, vol. 45, no. 22, pp. $704-709,2012$.

[8] S. Kim, S. Choi, and H. S. Kim, "Aerial manipulation using a quadrotor with a two dof robotic arm," in 2013 IEEE/RSJ International Conference on Intelligent Robots and Systems, 2013, pp. 4990-4995.

[9] G. Arleo, F. Caccavale, G. Muscio, and F. Pierri, "Control of quadrotor aerial vehicles equipped with a robotic arm," in 21 St mediterranean conference on control and automation. IEEE, 2013, pp. 1174-1180.

[10] F. Caccavale, G. Giglio, G. Muscio, and F. Pierri, "Adaptive control for uavs equipped with a robotic arm," IFAC Proceedings Volumes, vol. 47, no. 3, pp. $11049-11054,2014$.

[11] G. Antonelli and E. Cataldi, "Adaptive control of arm-equipped quadrotors. theory and simulations," in 22nd Mediterranean Conference on Control and Automation, 2014, pp. 1446-1451.

[12] R. Jiao, M. Dong, R. Ding, and W. Chou, "Control of quadrotor equipped with a two dof robotic arm," in 2018 3rd International Conference on Advanced Robotics and Mechatronics (ICARM). IEEE, 2018, pp. 437-442.

[13] S. Kannan, M. A. Olivares-Mendez, and H. Voos, "Modeling and control of aerial manipulation vehicle with visual sensor," IFAC Proceedings Volumes, vol. 46, no. 30, pp. 303 - 309, 2013.

[14] A. E. Jimenez-Cano, J. Martin, G. Heredia, A. Ollero, and R. Cano, "Control of an aerial robot with multi-link arm for assembly tasks," in 2013 IEEE International Conference on Robotics and Automation, 2013, pp. 4916-4921.

[15] G. Heredia, A. E. Jimenez-Cano, I. Sanchez, D. Llorente, V. Vega, J. Braga, J. A. Acosta, and A. Ollero, "Control of a multirotor outdoor aerial manipulator," in 2014 IEEE/RSJ International Conference on Intelligent Robots and Systems, 2014, pp. 3417-3422.

[16] S. Kannan, M. Alma, M. A. Olivares-Mendez, and H. Voos, "Adaptive control of aerial manipulation vehicle," in 2014 IEEE International Conference on Control System, Computing and Engineering (ICCSCE 2014), 2014, pp. 273-278.

[17] Y. Stergiopoulos, E. Kontouras, K. Gkountas, K. Giannousakis, and A. Tzes, "Modeling and control aspects of a uav with an attached manipulator," in 2016 24th Mediterranean Conference on Control and Automation (MED), June 2016, pp. 653-658.

[18] K. Gkountas, D. Chaikalis, and A. Tzes, "Force control design for a robot manipulator attached to a uav," IFAC-PapersOnLine, vol. 51, no. 30, pp. 548-553, 2018.

[19] C. Zhiyuan, L. Yanyang, S. Yanhua, C. Hongyu, W. Bin, H. Mingqi, and Y. Rao, "Fuzzy sliding mode control for rotorcraft aerial manipulator with extended state observer," in 2020 Chinese Automation Congress (CAC). IEEE, 2020, pp. 1710-1714.

[20] M. Wang, J. Qi, J. Kang, C. Wu, and Z. Wang, "Rotorcraft aerial manipulator control based on improved adrc," in 2020 39th Chinese Control Conference (CCC). IEEE, 2020, pp. 6744-6749.

[21] Y. Chen, W. Zhan, B. He, L. Lin, Z. Miao, X. Yuan, and Y. Wang, "Robust control for unmanned aerial manipulator under disturbances," Ieee Access, vol. 8, pp. 129869-129 877, 2020.

[22] N. Mimmo, A. Macchelli, R. Naldi, and L. Marconi, "Robust motion control of aerial manipulators," Annual Reviews in Control, vol. 49, pp. 230-238, 2020.
[23] N. Bulut, A. Turgut, and K. Aríkan, "Computed torque control of an aerial manipulation system with a quadrotor and a 2-dof robotic arm," 2019.

[24] H. T. M. N. ElKholy, "Dynamic modeling and control of a quadrotor using linear and nonlinear approaches," Master's thesis, School of Sciences and Engineering, The American University in Cairo, April 2014.

[25] R. M. Murray, Z. Li, and S. S. Sastry, A mathematical introduction to robotic manipulation. CRC press, 2017.

[26] W. Khalil, "Dynamic modeling of robots using recursive newton-euler techniques," in ICINCO2010, 2010.

[27] W. Khalil and E. Dombre, Modeling, identification and control of robots. Butterworth-Heinemann, 2004.

[28] E. Cataldi, G. Muscio, M. A. Trujillo, Y. Rodríguez, F. Pierri, G. Antonelli, F. Caccavale, A. Viguria, S. Chiaverini, and A. Ollero, "Impedance control of an aerial-manipulator: Preliminary results," in 2016 IEEE/RSJ International Conference on Intelligent Robots and Systems (IROS). IEEE, 2016, pp. 3848-3853.

[29] S. Kannan, S. Quintanar-Guzman, J. Dentler, M. A. Olivares-Mendez, and $\mathrm{H}$. Voos, "Control of aerial manipulation vehicle in operational space," in 2016 8th International Conference on Electronics, Computers and Artificial Intelligence (ECAI). IEEE, 2016, pp. 1-4.

[30] B. Siciliano, L. Sciavicco, L. Villani, and G. Oriolo, Robotics: modelling, planning and control. Springer Science \& Business Media, 2010.

[31] B. Siciliano and O. Khatib, Springer handbook of robotics 2nd Edition. springer, 2016.

[32] Y. Shtessel, C. Edwards, L. Fridman, and A. Levant, Sliding mode control and observation. Springer, 2014, vol. 10.

[33] V. I. Utkin, Sliding modes in control and optimization. Springer Science \& Business Media, 2013.

[34] E. Tahoumi, "New robust control schemes linking linear and sliding mode approaches," Ph.D. dissertation, École centrale de Nantes, 2019.

[35] F. Plestan, Y. Shtessel, V. Bregeault, and A. Poznyak, "New methodologies for adaptive sliding mode control," International journal of control, vol. 83, no. 9, pp. 1907-1919, 2010.

[36] J. Burton and A. S. Zinober, "Continuous approximation of variable structure control," International journal of systems science, vol. 17, no. 6, pp. $875-885,1986$. 